1 Morphology, ultrastructure, and molecular phylogeny of Aphelidium collabens sp. nov. (Aphelida), a 2 parasitoid of a green alga Coccomyxa sp.

4 Kensuke Seto ${ }^{\mathrm{a}, 1}$, Toshihiro Matsuzawa ${ }^{\mathrm{b}, 2}$, Hitoshi Kuno ${ }^{\mathrm{b}}$, Maiko Kagami $\mathrm{i}^{\mathrm{a}, 2}$

${ }^{a}$ Graduate School of Environment and Information Sciences, Yokohama National University, Tokiwadai 79-7,

Hodogaya, Yokohama 240-8501, Japan

8 b Advanced Research and Innovation Center, DENSO CORPORATION, Komenoki-cho, Nisshin-Shi, Aichi 470-0111, 9 Japan

10 1Present address; Department of Ecology and Evolutionary Biology, University of Michigan, 1105 North 11 University, Ann Arbor, Michigan 48109, United States

$12 \quad{ }^{2}$ Corresponding author; e-mail kagami-maiko-bd@ynu.ac.jp

(Maiko Kagami),

13 toshihiro.matsuzawa.j4x@jp.denso.com (Toshihiro Matsuzawa)

15 Running title: A new species of Aphelidium 
17 Aphelids (Aphelida) are intracellular parasitoids of algae and represent one of the early diverging or sister lineages of the kingdom Fungi. Although Aphelida is a small group, molecular phylogenetic analyses revealed that many environmental sequences belong to Aphelida, suggesting that aphelids are distributed worldwide; however, the extent of their diversity is unclear. Here, we investigated a novel aphelid culture APH2, that parasitizes the green alga Coccomyxa sp. APH2 produced posteriorly uniflagellate zoospores, a defining character of the genus Aphelidium. The residual body of APH2 was spherical in the mature plasmodium, but became amorphous during zoospore formation and collapsed after zoospore discharge, which has not been described for other Aphelidium species. Zoospores of APH2 possessed a striated rhizoplast that extended anteriorly from the kinetosome to the posterior end of the nucleus, and a microtubular root arising from the side of the kinetosome and lying almost parallel to the rhizoplast, both of which are unique among aphelid taxa. A molecular phylogenetic analysis based on the 18S rDNA sequences placed APH2 as sister lineage to all other known aphelid sequences. Based on these results, we describe this aphelid as a new species, Aphelidium collabens.

Keywords: Aphelida, Aphelidium, Coccomyxa, molecular phylogeny, parasitoid, ultrastructure

Introduction

34 Aphelids (the phylum Aphelida) are obligate intracellular parasitoids of green, yellow-green, and diatom algae

35 (Karpov et al. 2014a). The life cycle of aphelids is as follows (Karpov et al. 2014a): 1) a dispersal spore (posteriorly

36 flagellated zoospore or amoeboid cell without flagellum) encysts on the surface of a host cell; 2) the encysted spore

37 germinates, penetrates the host cell wall, and invades the host cytoplasm as a naked cell; 3) the parasitoid develops as a plasmodium and engulfs the host cytoplasm by phagocytosis; and 4) multinucleated plasmodium fills the host cell entirely, and divides to produce dispersal spores. The four aphelid genera are distinguished based on their dispersal spore and habitat (Karpov et al. 2014a, 2017). Three genera (Aphelidium, Amoeboaphelidium, and Paraphelidium) inhabit freshwater environments, while the monotypic genus Pseudaphelidium inhabits marine 
pseudopodia as dispersal spores (Karpov et al. 2014a), while Amoeboaphelidium produces amoeboid cells with or without immotile pseudocilium (Karpov et al. 2013, 2014a). Paraphelidium produces posteriorly uniflagellate zoospores but is distinguished from Aphelidium based on the characters of the pseudopodia (Karpov et al. 2017).

Molecular phylogenetic analysis revealed that aphelids are clustered with rozellids (Rozellomycota = Cryptomycota; herein, we use the former name according to Tedersoo et al. 2018) and Microsporidia (Karpov et al. 2013, Letcher et al. 2013). The so-called ARM (Aphelids-Rozellids-Microsporidia) clade (Karpov et al. 2013) was sister to traditional fungi (Ascomycota, Basidiomycota, Chytridiomycota sensu lato, and paraphyletic Zygomycota). Although the ARM clade was not strongly supported, it was described as the superphylum Opisthosporidia (Karpov et al. 2014a). Recently, Torruella et al. (2018) analyzed transcriptomes of Paraphelidium tribonematis and conducted phylogenomic analyses using several datasets of multiple protein markers or single-copy protein domains. The results showed that Opisthosporidia was paraphyletic, and that $P$. tribonematis was sister to the traditional fungi. Karpov et al. (2014a) accommodated aphelids in the new phylum Aphelida and regarded Opisthosporidia as not being a true fungus. By contrast, some authors (Berbee et al. 2017, Tedersoo et al. 2018) included aphelids, rozellids, and microsporidia within the kingdom Fungi. Although the taxonomic placement of aphelids remains to be determined, it is clear that aphelids along with rozellids and microsporidia are important lineages for understanding the early evolution of the Fungi and its defining characters (Richards et al. 2017). 
Light microscopy

71 The zoospore was posterior uniflagellate, ellipsoidal, $1.8-2.5 \mu \mathrm{m}$ in length, $1.0-1.3 \mu \mathrm{m}$ in width, and contained

72 a refractive inclusion (multiple lipid globules) in the apical region (Fig. 1A, B). The flagellum was 6.0-7.5 $\mu \mathrm{m}$

73 long and included an acroneme of 1.0-1.5 $\mu \mathrm{m}$. Amoeboid movement of zoospores was not observed.

74 Zoospores encysted on the surface of Coccomyxa sp. cells (Fig. 1C), germinated, penetrated the host cell wall 75 by means of a penetration tube, and invaded the host cytoplasm leaving an empty cyst (Fig. 1D). The parasitoid 76 developed as a phagotrophic amoeba in the host cell and engulfed the host cytoplasm. Host chloroplast was 77 divided into several fragments and decreased in size (Fig. 1E, F). During the development of parasitoid, an 78 orange-colored residual body was observed in the central region of the cell (Fig. 1E, F). Following its development, the parasitoid completely consumed the host chloroplast and developed into a plasmodium with a conspicuous, spherical residual body 1.7-2.3 $\mu \mathrm{m}$ in diameter (Fig. 1G). During zoospore formation, the residual body gradually collapsed (Fig. $1 \mathrm{H}, \mathrm{I})$. At the stage of zoospore discharge, the host cell swelled slightly (Fig. 1I) and a small pore developed in the host cell wall (Fig. 1M, arrowhead), from which zoospores were discharged (Fig. 1J-M). The discharged zoospores were spheroidal and stopped near the pore for several minutes (Fig. 1L). The zoospores became ellipsoidal and swam away (Fig. 1M). Several tiny fragments of residual body material were observed in the empty host cell (Fig. 1L, arrows). Empty cysts with a penetration tube remained on the surface of the empty host cell (Fig. 1N, O). The empty cyst was spherical and 1.0-1.5 $\mu \mathrm{m}$ in size. The penetration tube was up to $1.5 \mu \mathrm{m}$ in length.

\section{Transmission electron microscopy}

90 The zoospore was ellipsoidal with a single posterior flagellum (Fig. 2A, B). One or two filose pseudopodia extended from the posterior region (Fig. 2A, F), which were not visible under light microscopy. Multiple rounded lipid globules were present in the anterior region (Fig. 2A, B). An elongated nucleus was observed in 
94 mitochondria with flat cristae were evident in the posterior region (Fig. 2B-D). A kinetosome and

95 nonflagellated centriole ( $\mathrm{NfC}$ ) were aligned at an angle of $c a .60^{\circ}$ (Fig. $2 \mathrm{C}$ ). Although we could not find any transverse sections of $\mathrm{NfC}$, we identified the short cylindrical structure in $\mathrm{Fig} .2 \mathrm{C}$ as $\mathrm{NfC}$ because it had similar width as the kinetosome. A striated rhizoplast covered the anterior end of the kinetosome, extended anteriorly in the cytoplasm (Fig. 2A, C, D). The rhizoplast connected to the posterior end of the nucleus (Fig 2A, C) and was closely associated with the mitochondrion by lateral side (Fig 2D). A microtubular root composed of three microtubules extended from the side of the kinetosome to almost the anterior end of the zoospore body (Fig. 2B, D, I-K). Serial sections revealed that the kinetosome was composed of nine microtubule doublets (Fig. 2E$\mathrm{H}$ and $\mathrm{I}-\mathrm{K})$ and did not contain a cartwheel structure. A flagellum with a typical $9+2$ structure was observed in a transverse section (Fig. 2L). A general scheme of the zoospore ultrastructure is illustrated in Figure 3.

We observed parasitoids at several developmental stages in a host cell (Fig. 4). An empty cyst with a penetration tube was observed (Fig. 4A). At the early stage of parasitoid development, a host/parasitoid interface was visible (Fig. 4B, arrows). After the parasitoid almost filled the host cell, the host chloroplast was engulfed and divided into several fragments by the parasitoid. A residual body was observed in the parasitoid cell at this stage (Fig. 4C). The parasitoid consumed the host chloroplast, leaving several small chloroplast fragments at the periphery (Fig. 4D). The parasitoid developed into a multinucleate plasmodium (Fig. 4E). A food vacuole was located near the central region of the parasitoid cell and likely contained remnants of the digested host chloroplast (Fig. 4D, E). Mitochondrial cristae in the intracellular parasitoid exhibited predominately flat (Fig. 4A, G, H) or rarely tubular (Fig. 4I) profiles. The plasmodium divided to produce zoospores (Fig. 4F).

Molecular phylogenetic analysis

In the maximum-likelihood (ML) tree based on the $18 \mathrm{~S}$ rDNA sequences (Fig. 5), APH2 was placed in an independent position and was sister to the clade including all sequenced known taxa of aphelids and related 
119 bootstrap value $=72$, Bayesian posteriorly probability $=0.98$ ). Aphelidium desmodesmi and 120 Amoeboaphelidium occidentale formed a clade with high statistical support (ML bootstrap value $=96$, Bayesian 121 posteriorly probability = 1), and both Aphelidium and Amoeboaphelidium were polyphyletic. Aphelidium aff. 122 melosirae P-1 and Aphelidium tribonematis were sister to each other. Paraphelidium tribonematis and 123 Paraphelidium letcheri, together with related environmental sequences, formed a monophyletic clade.

\section{Taxonomy}

Aphelidium collabens K. Seto sp. nov. (ICN, International Code of Nomenclature for algae, fungi, and plants; 127 ICZN, International Code of Zoological Nomenclature)

Description: Endobiotic parasitoid of Coccomyxa sp., engulfing host cytoplasm by phagotrophy like amoeba, developing to a multinucleate plasmodium, and dividing into zoospores. Residual body orange-colored, spherical, 1.7-2.3 $\mu \mathrm{m}$ in diameter at later stages of plasmodial development, and gradually collapsing during zoospore formation. Zoospores discharged from a small pore that occurs in host cell wall. Zoospore ellipsoidal, 1.8-2.5 $\mu \mathrm{m}$ in length, 1.0-1.3 $\mu \mathrm{m}$ in width, having a single posterior flagellum, $6.0-7.5 \mu \mathrm{m}$ long, including an acroneme of 1.0-1.5 $\mu \mathrm{m}$. Amoeboid movement of zoospore not observed. Zoospore with multiple rounded lipid globules at anterior region; an elongated nucleus located at lateral region of the zoospore body; multiple rounded mitochondria with flat cristae located at posterior region; one or two filose pseudopodia occurring 136 from posterior region; ribosomes dispersed in the cytoplasm; kinetosome composed of microtubular doublets; 137 nonflagellated centriole lying at an angle of $c a .60^{\circ}$ to kinetosome; striated rhizoplast covering anterior end of 138 kinetosome and extending toward near the posterior end of nucleus; microtubular root composed of three 139 microtubules extending from the side of kinetosome to anterior region of the zoospore body. Zoospore cyst sessile or with a short stalk, 1.0-1.5 $\mu \mathrm{m}$ in diameter.

141 Type material: One plastic-embedded specimen (TNS-F-88796 holotype designated here) fixed for 142 transmission electron microscopic observation and deposited in the herbarium of the National Museum of 143 Nature and Science (TNS), Tokyo; culture APH2 (ex-type culture) isolated by K. Seto. 

Collection date: May 17, 2018.

Etymology: collabens $($ Latin) $=$ collapsing, referring to collapse of the residual body during zoospore 148 formation MycoBank: MB832009

\section{Discussion}

\section{Morphology}

154 The lifecycle of Ap. collabens culture APH2 is congruent with that of aphelids (Karpov et al. 2014a). Zoospore 155 encysts on the host cell surface and penetrates the host cell wall by means of a penetration tube. Parasitoid invades the host cell, engulfs the host cytoplasm by phagocytosis, and develops as a plasmodium. Finally, the multinucleate plasmodium divides to form zoospores.

The three freshwater-inhabiting aphelids are classified based on the features of zoospores (Karpov et al. 2014a). Aphelidium has motile and posteriorly uniflagellate zoospores, which can produce pseudopodia, while Amoeboaphelidium produces amoeboid cells with or without a pseudocilium instead of a flagellum. zoospores of Ap. collabens shows no amoeboid movement, which is observed in some described species; e.g., chlorococcorum f. majus (Gromov and Mamkaeva 1970, Scheffel 1925). Although the zoospore of Aphelidium 
not show the amoeboid movement (Gromov and Mamkaeva 1970). Fott (1957) provided an illustration of zoospores without pseudopodia in the description of Ap. chlorococcorum, but did not mention the amoeboid movement of zoospores. The zoospores of Ap. chlorococcorum f. majus are spherical and 3-4 $\mu \mathrm{m}$ in diameter,

172 in contrast to the ellipsoidal and smaller swimming zoospores of $A p$. collabens. The zoospores of $A p$. chaetophorae are spherical and immobile immediately after discharge and become oval when swimming away, thus resembling those of $A p$. collabens despite the difference in size. Although the zoospores of Ap. collabens shows no amoeboid movement, they possess filose pseudopodia at the posterior position. Similar posterior the penetration tube of zoospore cyst based on their TEM observation. In the present study, we revealed that collabens. 
196 Zoospore ultrastructure includes important taxonomic characteristics for classification of zoosporic fungi, 197 especially Chytridiomycota (Powell and Letcher 2014). In aphelids, detailed observation of zoospore 198 ultrastructure had been conducted only for Ap. chlorococcorum f. majus (Gromov and Mamkaeva 1975) and 199 Ap. desmodesmi (Letcher et al. 2017b). Karpov et al. (2019) performed detailed ultrastructural observations of 200 the zoospores of Ap. chlorococcorum f. majus, Ap. tribonematis, Ap. aff. melosirae, and P. tribonematis. They 201 focused on the flagellar apparatus (kinetid) structure and discussed its evolutionary trends. In the present 202 study, we observed the zoospore ultrastructure of $A p$. collabens in detail, enabling comparison with other 203 aphelid taxa.

204 The $\mathrm{NfC}$ is angled to the kinetosome in aphelid zoospores; the angle varies among taxa. It may be orthogonal in Ap. chlorococcorum f. majus, $30-45^{\circ}$ in Ap. aff. melosirae, or $30^{\circ}$ in Ap. tribonematis (Karpov et al. 2019). Letcher et al. (2017b) showed that the NfC of Ap. desmodesmi is parallel or at a slight angle to the kinetosome, but appears to be orthogonal or acutely angled to the kinetosome in their transmission electron micrographs (Fig. 3E in Letcher et al. 2017b) as mentioned by Karpov et al. (2019). In Ap. collabens, the NfC is about $60^{\circ}$ to the kinetosome, a more acute angle than in Ap. aff. melosirae and Ap. tribonematis.

The kinetosome of Ap. collabens is composed of nine doublet microtubules and does not contain a cartwheel structure. Paraphelidium tribonematis also possesses a kinetosome composed mainly of doublet microtubules (Karpov et al. 2019). Despite its reduced kinetosome, the zoospores of Ap. collabens and 213 P. tribonematis (Karpov et al. 2019) possess an axoneme with a typical $9+2$ structure and can actively swim 214 by beating of the flagellum. Based on the phylogenetic position of Ap. collabens and P. tribonematis, independent reduction of the kinetosome structure might have occurred during the evolution of aphelids.

Aphelidium collabens possesses a rhizoplast and microtubular root, both of which are associated with the 217 kinetosome and extend to the anterior region of the cytoplasm. Other aphelid taxa also have structures associated with the kinetosome (Karpov et al. 2019). Aphelidium chlorococcorum f. majus has the most complex kinetid structure (Gromov and Mamkaeva 1975, Karpov et al. 2019): a fibrillar root connecting the 
kinetosome, and microtubules occurring from the distal end of the basal foot. Aphelidium tribonematis lacks

222 a fibrillar root and basal foot but possesses a microtubule that emerges from the side of the kinetosome 223 (Karpov et al. 2019). Paraphelidium tribonematis possesses a fibrillar root, which is often, but not always, 224 connected to the mitochondrion, and two microtubules from the side of the kinetosome and the distal end of 225 the fibrillar root (Karpov et al. 2019). Aphelidium desmodesmi has no structure associated with the kinetosome 226 (Letcher et al. 2017b). The rhizoplast of Ap. collabens is most similar to the fibrillar root of Ap. chlorococcorum 227 f. majus and P. tribonematis because their position is congruent. However, the fibrillar root is not striated like 228 the rhizoplast of Ap. collabens; instead, it is uniformly electron dense (Karpov et al. 2019), and the rhizoplast 229 of Ap. collabens is not connected to the mitochondrion like the fibrillar root. Rozella spp. in Rozellomycota also 230 possess a rhizoplast that connects the anterior end of the kinetosome to the posterior end of the centrally 231 located mitochondrion (Held 1975, Letcher et al. 2017a, 2018). Furthermore, Rozella spp. have microtubules 232 that emerge from the side of the kinetosome and extend almost parallel to the rhizoplast, similar to $A p$. 233 collabens. Although the statistical support for the phylogenetic position of Ap. collabens was moderate, it was 234 placed in the sister branch to all other known and sequenced aphelid species in our molecular phylogenetic analysis. Karpov et al. (2019) pointed out that the rhizoplast (fibrillar root) between the kinetosome and mitochondrion is an ancestral character of Fungi and Opisthosporidia. Our finding of a striated rhizoplast in $A p$. collabens, together with its phylogenetic position, strengthens this hypothesis.

\section{Phylogeny and taxonomy of aphelids}

240 In contrast to prior reports (Karpov et al. 2019, Letcher et al. 2017b), Aphelidium and Amoeboaphelidium were not monophyletic in our phylogenetic analysis. In Letcher et al. (2017b), three species of Aphelidium-Ap. aff. melosirae, Ap. desmodesmi, and Ap. tribonematis-formed a monophyletic clade. However, Letcher et al. 243 (2017b) did not include many of the environmental sequences related to Aphelidium spp. and 244 Amoeboaphelidium spp. that were analyzed by Karpov et al. (2019), and in the present study. These environmental sequences might alter the tree topology. The difference between the results of Karpov et al. 
246 (2019) and our findings lies in the phylogenetic position of Ap. desmodesmi and Am. occidentale. These two 247 species were shown to be sister to each other in the present study, with strong statistical support. Recent 248 phylogenetic analysis (Tcvetkova et al. 2019) also demonstrated this relationship between Ap. desmodesmi and 249 Am. occidentale. In the phylogenetic tree of Karpov et al. (2019) and Tacvetkova et al. (2019), Ap. desmodesmi 250 was long-branched in comparison with our result. As mentioned in the Methods, a portion of the 18S rDNA 251 region and internal transcribed spacer (ITS) region are joined without gaps in Ap. desmodesmi. We eliminated 252 the sequences of the ITS and 28S rDNA regions prior to automatic alignment. Without this procedure, the ITS 253 region of $A p$. desmodesmi was improperly aligned with the latter portion of the $18 \mathrm{~S}$ rDNA sequences of other 254 taxa. Some preliminary phylogenetic analyses using this improper alignment (data not shown) yielded results 255 similar to those of Karpov et al. (2019); i.e., long branch of Ap. desmodesmi and separation of Ap. desmodesmi from Am. occidentale. Our molecular phylogeny indicates that two independent losses of the flagellum occurred in Am. protococcarum and Am. occidentale. However, the phylogenetic relationship of Am. protococcarum with Am. occidentale (+ Ap. desmodesmi) is unclear because the phylogenetic tree was poorly resolved. Phylogenetic analysis using multiple markers is needed to allow deeper discussion of the phylogeny of Amoeboaphelidium. Furthermore, the phylogenetic positions of the three other Amoeboaphelidium species described -Am. achnanthidis, parasitic on diatoms (Scheffel 1925); Am. chlorellavorum, parasitic on Chlorella (Gromov and Mamkaeva 1968); and Am. radiatum, parasitic on Ankistrodesmus and Kirchneriella (Gromov and Mamkaeva 1969)-must be clarified.

As with Amoeboaphelidium, Aphelidium was polyphyletic in the phylogenetic tree. It was separated into three lineages: Ap. collabens, Ap. desmodesmi, and Ap. aff. melosirae + Ap. tribonematis. Currently, the only defining character of Aphelidium is a posteriorly uniflagellate zoospore. Paraphelidium spp. also produce uniflagellate zoospores, but are distinguishable from Aphelidium based on the character of the pseudopodia (Karpov et al. 2017). The three lineages of Aphelidium revealed in the present study exhibited zoospores with different features. Aphelidium collabens has small $(1.8-2.5 \times 1.0-1.3 \mu \mathrm{m})$ and ellipsoidal zoospores without amoeboid movement. The zoospores of $A p$. desmodesmi are small $(1.6-1.9 \mu \mathrm{m})$ and possess multiple filose pseudopodia (Letcher et al. 2017b). The zoospores of Ap. aff. melosirae and Ap. tribonematis are larger than 
those of Ap. collabens and Ap. desmodesmi and produce short lobopodium (or lamellipodium) and one or more short filopodia (Karpov et al. 2014b, 2016). The zoospore ultrastructure is correlated with the phylogenetic lineages of Aphelidium. Aphelidium collabens possesses a rhizoplast, while Ap. desmodesmi and Ap. tribonematis do not (Letcher et al. 2017b, Karpov et al. 2019). The zoospore ultrastructure of $A p$. desmodesmi and Ap. tribonematis are similar, but the latter species has a microtubule arising from the side of the kinetosome (Karpov et al. 2019) while the former species does not. Although Ap. chlorococcorum f. majus has a more complex kinetid structure, its phylogenetic position is unknown because sequence data are not available (Karpov et al. 2019). A taxonomic revision of the genus Aphelidium is needed. However, the type species of Aphelidium, Ap. deformans parasitic on Coleochaete solula described by Zopf (1885), has not been examined using modern methods. Therefore, we tentatively describe APH2 as a new species of Aphelidium.

In conclusion, our characterization of Ap. collabens expands knowledge of the ultrastructural and phylogenetical diversity of aphelids. However, there were many environmental sequences in the phylogenetic tree, so aphelid diversity requires further investigation. Reexamination of known aphelid species and investigation of novel taxa are needed for taxonomic revision of Aphelida.

\section{Methods}

Isolation and culturing: Water samples were collected from an outdoor pond containing the green alga Coccomyxa sp. strain KJ, which was infected by an unknown aphelid. Strain KJ was established by Kyoto University and DENSO CORPORATION (Aichi, Japan) as part of the 'Research and Development for Production and Utilization of Renewable Energy in Rural Areas: Development of Technologies for Production of Alternative Fuel from Microalgae' project funded by the Ministry of Agriculture, Forestry, and Fisheries of Japan (Yoshimitsu et al. 2018). To establish a dual culture of the aphelid and its host, a single algal cell infected by the aphelid was isolated by micro-pipetting and transferred into a well of a microplate containing the host alga in Wright's cryptophyte (WC) medium (Guillard and Lorenzen 1972). The aphelid-alga dual culture (APH2) was maintained at $25^{\circ} \mathrm{C}$ in Erlenmeyer flasks aerated with $2 \%(\mathrm{v} / \mathrm{v}) \mathrm{CO}_{2}$ gas at a flow rate of $10 \mathrm{~mL} / \mathrm{min}$. The culture 
was maintained under a 12:12-hour light: dark cycle with irradiance at $100 \mu \mathrm{mol}$-photons $\cdot \mathrm{m}^{-2} \cdot \mathrm{s}^{-1}$ using a LEET20701-LD9 light (Toshiba Lighting \& Technology Corporation, Kanagawa, Japan).

Light microscopy: For morphological observations of APH2, 12-48-hour-old cultures were used. Living cultures mounted in WC medium were observed on slides. Zoospores and thalli on the host alga were imaged using an Axio Imager 2 microscope equipped with an Axiocam 512 color camera (Carl Zeiss, Tokyo, Japan).

Transmission electron microscopy: To observe zoospore ultrastructure, 48-hour-old culture containing many swimming zoospores was used. A zoospore suspension was generated by passing through a Minisart 1.2 $\mu \mathrm{m}$ filter (Sartorius, Tokyo, Japan). For fixation, the zoospore suspension was mixed with an equal volume of 2.5\% glutaraldehyde and $2 \%$ osmium tetroxide in WC medium (final concentrations, $1.25 \%$ glutaraldehyde and $1 \%$ osmium tetroxide). The mixture was incubated on ice for $90 \mathrm{~min}$, and the fixed zoospores were pelleted at $2,000 \mathrm{~g}$ at $0^{\circ} \mathrm{C}$ for $30 \mathrm{~min}$. After washing in distilled water, the pellet was embedded in $1.5 \%$ agarose (lowgelling temperature type VII-A; Sigma-Aldrich, Tokyo, Japan). Agarose blocks containing zoospores were dehydrated in an ethanol series $(10 \%, 30 \%, 50 \%, 70 \%, 75 \%$, and $90 \%$ for 15 min per step, and $95 \%$ once and 100\% twice for $20 \mathrm{~min}$ each) and embedded in Agar Low-Viscosity Resin (Agar Scientific, Stansted, UK). For ultrastructural observation of infection process, 12-, 36-, and 48-day-old cultures were prepared as described above. Ultrathin sections were prepared with an RMC MT-X ultramicrotome (RMC Products, Tucson, AZ, USA), and stained with platinum blue (Inaga et al. 2007) and lead citrate (Venable and Coggeshall 1965). The sections were imaged using an HT7700 transmission electron microscope (Hitachi, Tokyo, Japan) at an acceleration voltage of $80 \mathrm{kV}$.

DNA extraction, amplification, and sequencing: DNA was extracted from APH2 dual culture using a DNeasy PowerSoil Kit (Qiagen, Tokyo, Japan) according to the manufacturer's instructions. We amplified the 18S rDNA, ITS1-5.8S-ITS2, and 28S rDNA loci of aphelid by PCR using KOD FX Neo (Toyobo, Osaka, Japan) with the NS1 (White et al. 1990) and RCA95m (Wurzbacher et al. 2019) primers (the latter one is highly specific to fungi). The thermal cycling conditions for PCR amplification were as follows: $95^{\circ} \mathrm{C}$ for $5 \mathrm{~min}, 10$ cycles of denaturation at $98^{\circ} \mathrm{C}$ for $10 \mathrm{~s}$, annealing at $55-50^{\circ} \mathrm{C}\left(0.5^{\circ} \mathrm{C}\right.$ decrease per cycle) for $30 \mathrm{~s}$, and extension at $68^{\circ} \mathrm{C}$ for $5 \mathrm{~min}$, followed by 30 cycles of $98^{\circ} \mathrm{C}$ for $10 \mathrm{~s}, 50^{\circ} \mathrm{C}$ for $30 \mathrm{~s}$, and $68^{\circ} \mathrm{C}$ for $5 \mathrm{~min}$. The PCR products were purified by 
323 ExoSAP-IT (Thermo Fisher Scientific, Tokyo, Japan) and sequenced by the Fasmac sequencing service 324 (Kanagawa, Japan) using the following primers: NS1, NS4, NS6 (White et al. 1990), and NS8z (O'Donnell et al. 325 1998) for $18 S$ rDNA; and LROR (Rehner and Samuels 1994) and LR5 (Vilgalys and Hester 1990) for $28 S$ rDNA. 326 The obtained sequences were deposited in GenBank under the accession numbers LC488190 and LC488191.

Molecular phylogenetic analysis: We created a dataset of the $18 \mathrm{~S}$ rDNA sequences of Aphelida and Rozellomycota, including related environmental sequences. Nuclearia pattersoni and Nuclearia simplex were selected as outgroup taxa. The data of Ap. desmodesmi (GenBank Accession No.: KY249641) contained the sequences of the 18S rDNA, ITS1-5.8S-ITS2, and 28S rDNA loci. However, the preliminary sequence alignment between Ap. desmodesmi and other aphelid taxa indicated that part of the sequence of the 18S rDNA region (1,444 bases) and a part of or the entire ITS1-5.8S-ITS2 region of Ap. desmodesmi were joined without gaps. For this reason, we excluded the sequences of the ITS1-5.8S-ITS2 and 28S rDNA regions from the data of Ap. desmodesmi prior to the next procedure. Sequences were automatically aligned with MAFFT version 7.409 (Katoh and Standley 2013). Ambiguously aligned regions were excluded using trimAl version 1.2 (CapellaGutiérrez et al. 2009) with a gappyout model. The ML tree was inferred using RAxML version 8.2.7 (Stamatakis 2014). We ran an analysis using the GTR + GAMMA + I model, and applied the "-fa" option to conduct a rapid bootstrap analysis with 1,000 replicates combining 200 searches for the optimal tree. A Bayesian analysis was run using MrBayes version 3.2.6 (Ronquist et al. 2012) and the GTR + GAMMA + I model with 5 million generations and sampling every 100 generations. The first $25 \%$ of trees were discarded as burn-in. Bayesian

Acknowledgments

This work was supported by the Japan Society for the Promotion of Science KAKENHI (grant no. 16H02943).

Berbee ML, James TY, Strullu-Derrien C (2017) Early diverging fungi: Diversity and impact at the dawn of 
Capella-Gutiérrez S, Silla-Martínez JM, Gabaldón T (2009) TrimAl: a tool for automated alignment trimming in large-scale phylogenetic analyses. Bioinformatics 25:1972-1973

Fott B (1957) Aphelidium chlorococcarum spec. nova, ein neuer Parasit in Grünalgen. Biologica (Santiago) 3:229-237

Gromov BV, Mamkaeva KA (1968) Amoeboaphelidium protococcarum sp. n. and Amoeboaphelidium chlorellavorum sp. n.-endoparasites of protococcous algae. Acta Protozool 6:221-225

Gromov BV, Mamkaeva KA (1969) The culture of Amoeboaphelidium radiatum sp. nov.-endoparasite of protococcous alga. Vestn Leningr Univ Ser Biol 9:140-144

Gromov BV, Mamkaeva KA (1970) The culture of Aphelidium chlorococcarum f. majus f. nova. Acta Protozool 7:263-267

Gromov BV, Mamkaeva KA (1975) Ultrastructure of zoospores of Aphelidium chlorococcarum Fott. Mikol Fitopatol 9:190-193

Guillard RRL, Lorenzen CJ (1972) Yellow-green algae with chlorophyllide C1,2. J Phycol 8:10-14

Held AA (1975) The zoospore of Rozella allomycis: ultrastructure. Can J Bot 53:2212-2232

Inaga S, Katsumoto T, Tanaka K, Kameie T, Nakane H, Naguro T (2007) Platinum blue as an alternative to uranyl acetate for staining in transmission electron microscopy. Arch Histol Cytol 70:43-49

Karpov SA, Cvetkova VS, Annenkova NV, Vishnyakov AE (2019) Kinetid structure of Aphelidium and Paraphelidium (Aphelida) suggests the features of the common ancestor of Fungi and Opisthosporidia. J Eukaryot Microbiol 66:911-924

Karpov SA, Mamkaeva MA, Aleoshin VV, Nassonova E, Lilje O, Gleason FH (2014a) Morphology, phylogeny, and ecology of the aphelids (Aphelidea, Opisthokonta) and proposal for the new superphylum Opisthosporidia. Front Microbiol 5:112

Karpov SA, Mamkaeva MA, Benzerara K, Moreira D, López-García P (2014b) Molecular phylogeny and ultrastructure of Aphelidium aff. melosirae (Aphelida, Opisthosporidia). Protist 165:512-526

Karpov SA, Mamkaeva MA, Moreira D, López-García P (2016) Molecular phylogeny of Aphelidium tribonemae 
reveals its sister relationship with $A$. aff. melosirae (Aphelida, Opisthosporidia). Protistology 10:97103

Karpov SA, Mikhailov KV, Mirzaeva GS, Mirabdullaev IM, Mamkaeva KA, Titova NN, Aleoshin VV (2013) Obligately phagotrophic aphelids turned out to branch with the earliest-diverging fungi. Protist 164:195-205

Karpov SA, Tcvetkova VS, Mamkaeva MA, Torruella G, Timpano H, Moreira D, Mamanazarova KS, LópezGarcía P (2017) Morphological and genetic diversity of Opisthosporidia: New aphelid Paraphelidium tribonemae gen. et sp. nov. J Eukaryot Microbiol 64:204-212

Katoh K, Standley DM (2013) MAFFT Multiple Sequence Alignment Software Version 7: Improvements in performance and usability. Mol Biol Evol 30:772-780

Letcher PM, Longcore JE, James TY, Leite DS, Simmons DR, Powell MJ (2018) Morphology, ultrastructure, and molecular phylogeny of Rozella multimorpha, a new species in Cryptomycota. J Eukaryot Microbiol 65:180-190

Letcher PM, Longcore JE, Quandt CA, Leite D da S, James TY, Powell MJ (2017a) Morphological, molecular, and ultrastructural characterization of Rozella rhizoclosmatii, a new species in Cryptomycota. Fungal Biol 121:1-10

Letcher PM, Lopez S, Schmieder R, Lee PA, Behnke C, Powell MJ, McBride RC (2013) Characterization of Amoeboaphelidium protococcarum, an algal parasite new to the Cryptomycota isolated from an outdoor algal pond used for the production of biofuel. PLoS ONE 8:e56232

Letcher PM, Powell MJ (2019) A taxonomic summary of Aphelidiaceae. IMA Fungus 1:4

Letcher PM, Powell MJ, Lee PA, Lopez S, Burnett M (2017b) Molecular phylogeny and ultrastructure of Aphelidium desmodesmi, a new species in Aphelida (Opisthosporidia). J Eukaryot Microbiol 64:655667

O’Donnell K, Cigelnik E, Benny GL (1998) Phylogenetic relationships among the Harpellales and Kickxellales. Mycologia 90:624-639

Powell MJ, Letcher PM (2014) Chytridiomycota, Monoblepharidomycotaand, and Neocallimastigomycota. In 
McLaughlin DJ, Spatafora JW (eds) The Mycota VII, Systematics and Evolution, Part A. Springer-Verlag, Berlin, pp 141-175

Rehner SA, Samuels GJ (1994) Taxonomy and phylogeny of Gliocladium analysed from nuclear large subunit ribosomal DNA sequences. Mycol Res 98:625-634

Richards TA, Leonard G, Wideman JG (2017) What Defines the "Kingdom" Fungi? In Heitman J, Howlett BJ, Crous PW, Stukenbrock EH, James TY, Gow NAR (eds) The Fungal Kingdom. John Wiley \& Sons, Ltd, pp $55-77$

Ronquist F, Teslenko M, van der Mark P, Ayres DL, Darling A, Höhna S, Larget B, Liu L, Suchard MA, Huelsenbeck JP (2012) MrBayes 3.2: Efficient Bayesian phylogenetic inference and model choice across a large model space. Syst Biol 61:539-542

Scheffel A (1925) Endophytische Phycomyceten-Parasiten der bacilleriaceen und einige neue Monadinen. Arch Für Protistenkd 52:1-141

Schweikert M, Schnepf E (1996) Pseudaphelidium drebesii, gen et spec nov (incerta sedis), a parasite of the marine centric diatom Thalassiosira punctigera. Arch Für Protistenkd 147:11-17

Stamatakis A (2014) RAxML version 8: a tool for phylogenetic analysis and post-analysis of large phylogenies. Bioinformatics 30:1312-1313

Tcvetkova VS, Zorina NA, Mamkaeva MA, Karpov SA (2019) Molecular phylogeny of Aphelidium arduennense sp. nov. - new representative of Aphelida (Opisthosporidia). Protistology 13:192-198

Tedersoo L, Sánchez-Ramírez S, Kõljalg U, Bahram M, Döring M, Schigel D, May T, Ryberg M, Abarenkov K (2018) High-level classification of the Fungi and a tool for evolutionary ecological analyses. Fungal Divers 90:135-159

Torruella G, Grau-Bové X, Moreira D, Karpov SA, Burns JA, Sebé-Pedrós A, Völcker E, López-García P (2018) Global transcriptome analysis of the aphelid Paraphelidium tribonemae supports the phagotrophic origin of fungi. Commun Biol 1:231

Venable JH, Coggeshall R (1965) A simplified lead citrate stain for use in electron microscopy. J Cell Biol 25:407-408 
Vilgalys R, Hester M (1990) Rapid genetic identification and mapping of enzymatically amplified ribosomal DNA from several Cryptococcus species. J Bacteriol 172:4238-4246

White TJ, Bruns T, Lee S, Taylor JL (1990) Amplification and direct sequencing of fungal ribosomal RNA genes for phylogenetics. In Innis MA, Gelfand DH, Sninsky JJ, White TJ (eds) PCR Protocols: A Guide to Methods and Applications. Academic Press, New York, pp 315-322

Wurzbacher C, Larsson E, Bengtsson-Palme J, Wyngaert SV den, Svantesson S, Kristiansson E, Kagami M, Nilsson RH (2019) Introducing ribosomal tandem repeat barcoding for fungi. Mol Ecol Resour 19:118127

Yoshimitsu Y, Abe J, Harayama S (2018) Cas9-guide RNA ribonucleoprotein-induced genome editing in the industrial green alga Coccomyxa sp. strain KJ. Biotechnol Biofuels 11:326

Zopf W (1885) Zur Morphologie und Biologie der niederen Pilzthiere (Monadinen), zugleich ein Beitrag zur Phytopathologie. Verlag von Veit \& Comp, Leipzig

\section{Figure Legends}

Figure 1. Morphology of Aphelidium collabens APH2 on host Coccomyxa sp. KJ. A, B. Zoospore. C. Encysted zoospore. D. Empty cyst. E, F. Development of a parasitoid inside a host cell. G. Mature parasitoid with a residual body. H-M. Zoospore formation and discharge. $\mathbf{N}, \mathbf{O}$. Empty host and attached empty cyst of a parasitoid. Scale bar $=5 \mu \mathrm{m} . \mathrm{Ac}=$ acroneme, $\mathrm{EC}=$ empty cyst, $\mathrm{Fl}=$ flagellum, $\mathrm{L}=$ lipid, $\mathrm{PT}=$ penetration tube. Arrows in L indicate fragments of residual body. Arrowhead in $\mathrm{M}$ indicates a small pore of the host cell wall.

Figure 2. Zoospore ultrastructure of Aphelidium collabens APH2. A, B. Longitudinal section of a zoospore. C. Longitudinal section of a kinetosome, nonflagellated centriole, and rhizoplast. D. Longitudinal section of a kinetosome, microtubular root, and rhizoplast. E-H. Transverse serial sections of the kinetosomal region. I-K. Transverse serial sections of a kinetosome and microtubular root. L. Transverse section of a flagellum. Scale bars $=0.5 \mu \mathrm{m}(\mathrm{A}, \mathrm{B}), 0.2 \mu \mathrm{m}(\mathrm{C}, \mathrm{D}, \mathrm{E}$ in $\mathrm{E}-\mathrm{H}$; I in $\mathrm{I}-\mathrm{K}, \mathrm{L}) . \mathrm{FP}=$ filose pseudopodia, $\mathrm{K}=$ kinetosome, $\mathrm{L}=$ lipid 
globule, $\mathrm{MR}=$ microtubular root, $\mathrm{Mt}=$ mitochondrion, $\mathrm{N}=$ nucleus, $\mathrm{NfC}=$ nonflagellated centriole, $\mathrm{Rh}=$ rhizoplast, $\mathrm{V}=$ vacuole. Arrows in $\mathrm{I}-\mathrm{K}$ indicate microtubules.

Figure 3. Schematic of the zoospore ultrastructure of Aphelidium collabens APH2. A. Longitudinal section through the zoospore. B. Longitudinal section of the kinetosome and nonflagellated centriole. C. Transverse section of the anterior end of the kinetosome and microtubular root. Abbreviations are as for Figure 2.

Figure 4. Ultrastructure of developmental stages of Aphelidium collabens APH2. A. Infected host cell with an Almost-mature plasmodium with multiple nuclei and a food vacuole. F. Zoospores produced in a host cell. G. Large image of mitochondria in D indicated by square. H. Large image of mitochondria in the other parasitoid cell. I. Large image of mitochondria in $C$ indicated by square. Scale bars $=1 \mu \mathrm{m}(\mathrm{A}-\mathrm{F}), 0.5 \mu \mathrm{m}(\mathrm{G}-\mathrm{I})$. $\mathrm{P}=$ parasitoid, $\mathrm{PT}=$ penetration tube, $\mathrm{RB}=$ residual body. Arrows in $\mathrm{B}$ indicate the host-parasite interface.

Figure 5. Maximum likelihood (ML) tree of Aphelida and Rozellomycota based on $18 \mathrm{~S}$ rDNA sequences. The tree was rooted with two Nuclearia spp. GenBank accession numbers of the operational taxonomic units are shown in parentheses. ML bootstrap support (MLBP) $\geq 50 \%$ and Bayesian posterior probabilities (BPP) $\geq 0.95$ are indicated as MLBP/BPP on the branches. Hyphens indicate MLBP values of $<50 \%$ or BPP values of $<0.95$. 